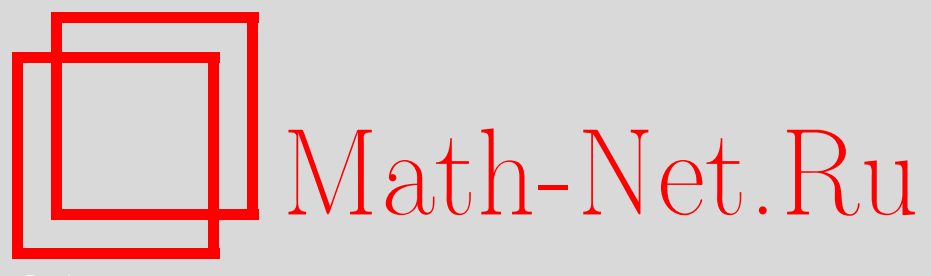

Н. Г. Кружилин, Обобщение теоремы Кернера о собственных отображениях, УМН, 2010, том 65, выпуск $6,187-188$

DOI: https://doi.org/10.4213/rm9399

Использование Общероссийского математического портала Math-Net.Ru подразумевает, что вы прочитали и согласны с пользовательским соглашением http://www . mathnet.ru/rus/agreement

Параметры загрузки:

IP : 54.198 .187 .58

26 апреля 2023 г., 11:23:31

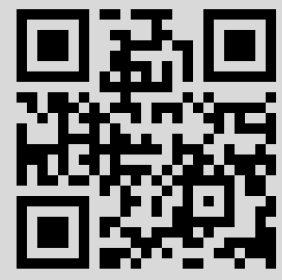




\section{Обобщение теоремы Кернера о собственных отображениях}

\section{Н. Г. Кружилин}

Голоморфное отображение

$$
f: D_{1} \rightarrow D_{2}
$$

между комплексными многообразиями называется собственным, если для всякого компактного подмножества $K \subset D_{2}$ его полный прообраз $f^{-1}(K)$ является компактным подмножеством $D_{1}$. Мы будем рассматривать случай многообразий одинаковой размерности. В этом случае, согласно известной теореме Реммерта, $f$ является конечным разветвленным накрытием: существует такое аналитическое подмножество $S \subset D_{2}$, вне которого отображение

$$
f: D_{1} \backslash f^{-1}(S) \rightarrow D_{2} \backslash S
$$

представляет собой конечное накрытие.

Вскоре после публикации результата Реммерта Г. Кернер [1] установил следующую теорему о продолжении для собственных отображений.

Теорема 1. Пусть $D_{1}$ и $D_{2}$ - римановы области над многообразиями Штейна одинаковой размерности $n \geqslant 2$, а $\widehat{D}_{1}$ u $\widehat{D}_{2}-u x$ оболочки голоморфности. Тогда всякое собственное голоморфное отображение

$$
f: D_{1} \rightarrow D_{2}
$$

продолжается до собственного голоморфного отображения

$$
\hat{f}: \widehat{D}_{1} \rightarrow \widehat{D}_{2} \text {. }
$$

В настоящей заметке доказывается результат, обобщающий теорему Кернера.

Теорема 2. Пусть $D_{1}$ - подобласть многообразия Штейна, $\widehat{D}_{1}$ - ее оболочка голоморфности, а $D_{2}-$ комплексное многообразие той же размерности $n \geqslant 2$. Пусть определено собственное голоморфное отображение

$$
f: D_{1} \rightarrow D_{2} .
$$

Тогда существуют пространство Штейна

$$
\widehat{D}_{2} \supset D_{2},
$$

являющееся оболочкой голоморфности $\mathrm{D}_{2}$, и собственное голоморфное отображение

$$
\hat{f}: \widehat{D}_{1} \rightarrow \widehat{D}_{2},
$$

продолжающее $f$.

ДокАзАТЕЛЬСтво. Пусть $k$ - кратность отображения $f$. В декартовом произведении $D_{1} \times D_{1}$ рассмотрим аналитическое подмножество

$$
S=\{(x, y) \mid f(x)=f(y)\} .
$$

Точке из первой компоненты произведения поставим в соответствие лежащие над ней точки $S$. В силу собственности $f$ мы получаем $k$-значное голоморфное соответствие. Считая образ отображения $D \subset \widehat{D}_{1}$ вложенным в комплексное линейное

Работа выполнена при поддержке программы "Ведущие научные школы" (грант НШ-3476.2010.1) и РФФИ. 
пространство и рассматривая симметрические функции от координат компонент этого соответствия, мы стандартным образом продолжаем его на $\widehat{D}_{1}$ до голоморфного соответствия $F$, образ которого будет лежать в $\widehat{D}_{1}$. График Г соответствия $F$ является аналитическим подмножеством $\widehat{D}_{1} \times \widehat{D}_{1}$. Очевидно, что $Г$ содержит диагональ в $\widehat{D}_{1} \times \widehat{D}_{1}$.

Теперь в $D_{1} \times D_{1} \times D_{1}$ рассмотрим аналитическое подмножество $\Lambda=\{(x, y, z)$ : $y \in F(x), z \in F(y)\}$. Это график $k^{2}$-значного голоморфного соответствия, продолжающегося до голоморфного соответствия $L: \widehat{D}_{1} \rightarrow \widehat{D}_{1} \times \widehat{D}_{1}$, из которого $F$ получается проекцией на первый сомножитель образа. Заметим, что, в силу определения $\Lambda$, над $D_{1}$ это множество инвариантно относительно действия симметрической группы $S_{3}$, а значит, оно и вообще $S_{3}$-инвариантно.

Для $x_{1}, x_{2} \in \widehat{D}_{1}$ будем писать $x_{1} \simeq x_{2}$, если пара $\left(x_{1}, x_{2}\right)$ лежит на $Г$. В силу отмеченной выше $S_{3}$-инвариантности это отношение симметрично и транзитивно, а значит, мы получили отношение эквивалентности на $\widehat{D}_{1}$.

Обозначив через $\infty$ несобственный элемент при одноточечной компактификации $\widehat{D}_{1}$, нетрудно видеть, что $F(z) \rightarrow \infty$ при $\widehat{D}_{1} \ni z \rightarrow \infty$. Действительно, если для некоторой последовательности $z_{k}$ существуют точки $y_{k} \in F\left(z_{k}\right)$ такие, что $y_{k} \nrightarrow \infty$, то мы можем считать, что $y_{k} \rightarrow y_{0} \in \widehat{D}_{1}$. Соответственно, $z_{k} \in F\left(y_{k}\right)$, но $\lim z_{k} \notin \widehat{D}_{1}$, хотя $y_{k} \rightarrow y_{0} \in \widehat{D}_{1}$, что противоречит определению $F$ как голоморфного соответствия $\widehat{D}_{1} \rightarrow \widehat{D}_{1}$.

Таким образом, $\simeq$ является собственным аналитическим отношением эквивалентности и мы можем определить фактор $\widehat{D}_{1} / \simeq$. Снова рассматривая вложение $\widehat{D}_{1}$ в комплексное линейное пространство, мы видим, что симметрические функции от координат прообразов точек из $\widehat{D}_{1} / \simeq$ корректно определены на факторпространстве и разделяют точки на нем. По теореме А. Картана [2], $\widehat{D}_{1} / \simeq$ оказывается комплексным аналитическим пространством. Нетрудно видеть, что оно является пространством Штейна. Соответствующая проекция - собственное отображение, которое естественно считать продолжением отображения $f$.

Заметим, что (в силу симметрии отношения $\simeq) F\left(\widehat{D}_{1} \backslash D_{1}\right)$ лежит в $\widehat{D}_{1} \backslash D_{1}$. Таким образом, $D_{2}$ вложено в пространство Штейна $\widehat{D}_{1} / \simeq$. Всякая голоморфная функция $g$ на $D_{2}$ поднимается до голоморфной функции на $D_{1}$ и, следовательно, продолжается до голоморфной функции на $\widehat{D}_{1}$, причем в силу аналитичности $\simeq$ ее значения в эквивалентных точках совпадают, так что $g$ продолжается до голоморфной функции на $\widehat{D}_{1} / \simeq$. Таким образом, $\widehat{D}_{1} / \simeq$ является оболочкой голоморфности $\widehat{D}_{2}$, что и завершает доказательство.

\section{Список литературы}

[1] H. Kerner, Arch. Math., 11 (1960), 44-49. [2] А. Картан, Комплексные пространства, сб. перев., Мир, М., 1965; пер. с англ.: Н. Cartan, Contributions to function theory, Internat. Colloq. Function Theory (Bombay, 1960), Tata Institute of Fundamental Research, Bombay, 1960, $1-15$.

Н. Г. Кружилин (N. G. Kruzhilin)

Математический институт им. В. А. Стеклова РАН

E-mail: kruzhil@mi.ras.ru
Представлено С. Ю. Немировским Принято редколлегией 23.11.2010 